Communications in Physics, Vol. 30, No. 4 (2020), pp. 311-318

DOI:10.15625/0868-3166/30/4/15081

\title{
EXPERIMENTAL VERIFICATION OF A THz MULTI-BAND METAMATERIAL ABSORBER
}

\section{TRAN VAN HUYNH ${ }^{1,2,3}$, BUI XUAN KHUYEN ${ }^{1,2}$, BUI SON TUNG ${ }^{1,2}$, VU DINH LAM ${ }^{2}$, LE THI HONG HIEP ${ }^{4}$ AND NGUYEN THANH TUNG ${ }^{1,2, \dagger}$}

${ }^{1}$ Institute of Materials Science, Vietnam Academy of Science and Technology, 18 Hoang Quoc Viet, Cau Giay, Hanoi, Vietnam

${ }^{2}$ Graduate University of Science and Technology, Vietnam Academy of Science and Technology, 18 Hoang Quoc Viet, Cau Giay, Hanoi, Vietnam Vietnam

${ }^{3}$ Department of Basic Sciences, University of Fire Prevention and Fighting, 243 Khuat Duy Tien, Thanh Xuan, Hanoi, Vietnam

${ }^{4}$ Department of Fundamental of Fire Fighting and Prevention, University of Fire Prevention and Fighting, 243 Khuat Duy Tien, Thanh Xuan, Hanoi, Vietnam

${ }^{\dagger}$ E-mail: tungnt@ims.vast.ac.vn

Received 20 May 2020

Accepted for publication 7 July 2020

Published 20 October 2020

\begin{abstract}
Multi-band metamaterial absorbers have been of great interest owing to their potentials for a wide range of communicating, sensing, imaging, and energy harvesting applications. In this work, we experimentally investigate a four-band metamaterial absorber operating at $\mathrm{THz}$ frequencies. The metamaterials are fabricated using the maskless UV photolithography and ebeam evaporation techniques. The absorption spectra of the proposed absorber are measured using the micro-Fourier transformed infrared spectroscopy. It was demonstrated that multi-band absorption behavior originates from different individual metamaterial resonators. The thickness of the dielectric spacer plays a key role in optimizing the absorption performance, in line with the predicted results on single-band $\mathrm{THz}$ absorbers.
\end{abstract}

Keywords: metamaterial absorbers, multi-band, $\mathrm{THz}$ frequencies.

Classification numbers: 81.05.Xj; 78.67.Pt; 71.20.Ps; 71.20.-b.

C2020 Vietnam Academy of Science and Technology 


\section{INTRODUCTION}

$\mathrm{THz}$ frequencies are usually considered from 0.1 to several ten $\mathrm{THz}$ (wavelengths of $3 \mathrm{~mm}$ to several $\mu \mathrm{m}$ ). Historically, the terahertz frequency band is often referred to as "the THz gap", where practical technologies for generating and detecting the radiation has not been developed efficiently and feasibly [1]. Since the late $20^{\text {th }}$ century, investigation has resulted in improved $\mathrm{THz}$ emitters and detectors, leading to intensified research in this area, especially for novel materials that interact with $\mathrm{THz}$ radiation in a desired manner [2].

In this regard, metamaterials (MMs) appear as one of the most charming candidates for $\mathrm{THz}$ materials [3]. While the electromagnetic properties of conventional materials essentially depend on their chemical compositions rather than on the sample size, MMs that are made up by periodically arranged sub-wavelength metallic structures, reveal a very unnatural but fascinating picture $[4,5]$. The properties of MMs are not only governed by the properties of the component materials but also by the geometrical features of the unit cells. Since most natural materials are not able to effectively interact with these electromagnetic waves in the THz range, MMs become a promising platform so to enhance the control in the light-matter interaction. In addition, in the $\mathrm{THz}$ frequency range, fabrication of subwavelength structures can be achieved in large (i.e. centimeter) scales using existing lithographic techniques [6].

While absorption losses are a drawback for $\mathrm{THz}$ applications that desire a high transmitivity, the high absorptivity is an important advance for those relying on electromagnetic radiation probing technologies $[7,8]$. For this reason, the concept of metal-structure/dielectric-substrate/metalmirror metamaterial absorbers (MMA) has been introduced [9] and has been further broadening the significance of metamaterials [10]. Geometry-unrestricted structures are being considered for metamaterial applications, starting from simple ones such as cut wire pair and split ring resonators [11] to more complex ones like Jerusalem, helical, or snowflake structures [12]. A drawback of these designs is that the obtained absorption bandwidth is too narrow to be useful for different sources of energy, thereby limiting the overall efficiency of the devices. Many computational studies therefore have proposed diversifying solutions for the broadband or multi-band MMAs [13-16]. The most practical approach is to stack different individual resonators in one joint unit cell. Unfortunately, the number of experimental efforts to fabricate and characterize the multi-band absorption behavior following the joint individual resonator approach has been very limited so far.

In this study, we experimentally investigate the multi-band absorption behavior of a $\mathrm{THz}$ MMA. Firstly, the individual MMA, which responds for a single-band absorption, is discussed to propose a four-band absorber design at around 50-60 THz. The four-band metamaterial absorbers are then fabricated using the maskless UV photolithography and e-beam evaporation techniques. Their absorption behavior is investigated using the micro-Fourier transformed infrared spectroscopy.

\section{EXPERIMENT}

Figure 1(a) describes the structure of the metamaterial consisting of resonators that are placed on top of a gold plate, separated by a $\mathrm{SiO}_{2}$ dielectric spacer. $\mathrm{SiO}_{2}$ is chosen because of its high compatibility in the micro-fabrication technologies and excellent transparency at $\mathrm{THz}$ range. The periodicities in the lateral $(E$ and $H$ ) directions are $2.5 \mu \mathrm{m}$ while the thicknesses of 
$\mathrm{Au}$ resonators and the ground plate are fixed at $100 \mathrm{~nm}$, respectively. The $\mathrm{SiO}_{2}$ dielectric spacer $t_{s}$ will be varied in our study. The excitation waves are unpolarized and perpendicular to the sample plane.

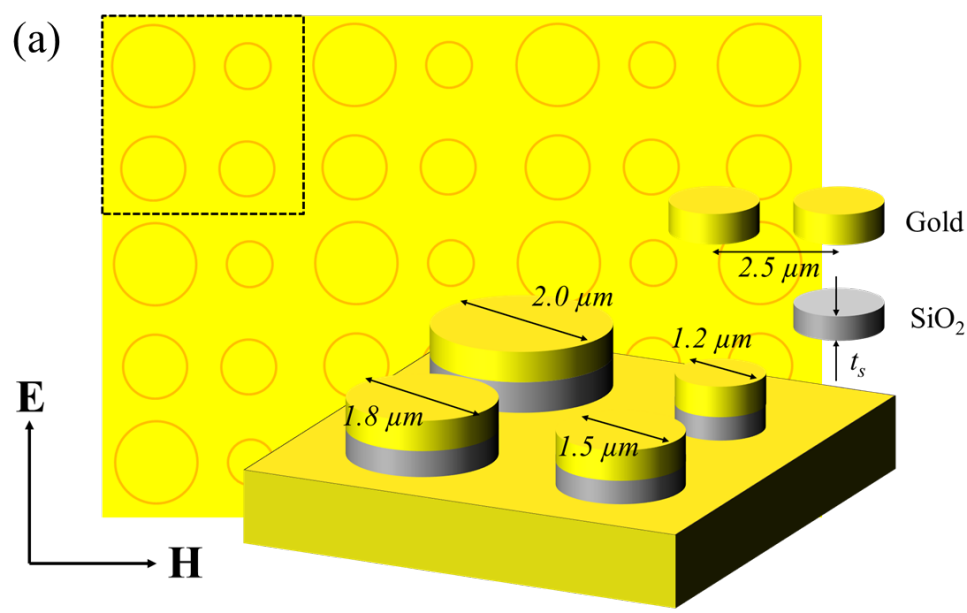

(b)

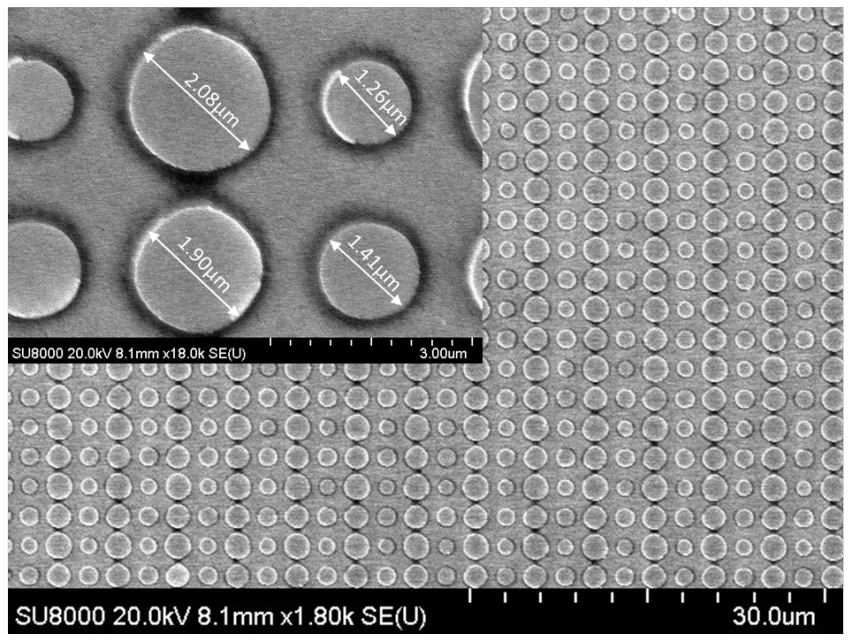

Fig. 1. (a) The schematic drawing of the four-band MMA structure and (b) the SEM images of the fabricated four-band MMA sample.

The MMA samples are fabricated using a three-step process. In the first step, a 100-nm $\mathrm{Au}$ film is deposited onto a $350-\mu \mathrm{m}$ silicon wafer right after a 5-nm $\mathrm{Cr}$ adhesion layer using the electron-beam evaporator. After that, an array of photoresist with disk-shaped structures is patterned on the Au film surface using the maskless UV-lithography (DL1000, Nano System Solutions). This technique allows large-scaled MMAs to be conveniently fabricated with an area up to a few squares of centimeter [10]. The final step is to deposit $\mathrm{SiO}_{2}$ and $\mathrm{Au}$ films followed by the lift-off process, forming the final structure as $\mathrm{Au}$ plate/ $/ \mathrm{SiO}_{2}$ disk/Au disk. Fig. 1(b) shows the scanning electron microscope (SEM) images of the fabricated four-band sample. 
The absorption property of the MMA is examined by a micro Fourier-transformed infrared spectrometer $(\mu$-FTIR 6300FV, Jasco). To achieve a good signal-to-noise ratio of IR signals, the sample chamber is filled with nitrogen gas. A liquid nitrogen-cooled high-sensitive MCT ( $\mathrm{HgCdTe})$ detector is used with a frequency resolution of $2 \mathrm{~cm}^{-1}$. The absorption defined as $A=1-T-R$ can be calculated from measured transmission and reflection. Due to the presence of the thick Au film, the transmission is blocked and the absorption can be obtained directly via the reflectance measurement. The reflection of samples is calibrated with that of a bare Au film. The disk diameters of fabricated samples are $1.20 \pm 0.1,1.50 \pm 0.1,1.80 \pm 0.1$ and $2.0 \pm 0.1 \mu \mathrm{m}$.

\section{RESULTS AND DISCUSSION}

\section{III.1. Single-band MMAs and the equivalent circuit model}

Single-band MMAs have been extensively studied by numerical calculations, identifying their frequency scalability, functional tunability, parametric dependence, and geometry diversity. THz single-band MMAs were also fabricated and experimentally characterized [6]. Under the electromagnetic excitation, the antiparallel electric currents are magnetically induced in the MMA metallic structures $[17,18]$. In this context, the MMA can be described as an electromagnetic $L C$ resonator and its resonant frequency, which corresponds to the absorption frequency, can be predicted by the equivalent circuit model. In general, the equivalent circuit model can be quantitatively constructed by analyzing the resonator geometrical parameters and the induced-charge distribution. This approach has been demonstrated as one of the most effective methods to describe the electromagnetic response of different kinds of MMs [19-22].

Figure 2 presents (a) the schematic drawing of the current distribution at the resonant frequency and (b) the equivalent circuit model for the single-band MMA. For the single-band MMAs, it has been understood that the charge currents are stimulated between the metallic resonators and their images on the metallic plate as depicted in Fig. 2(a) [17-22].

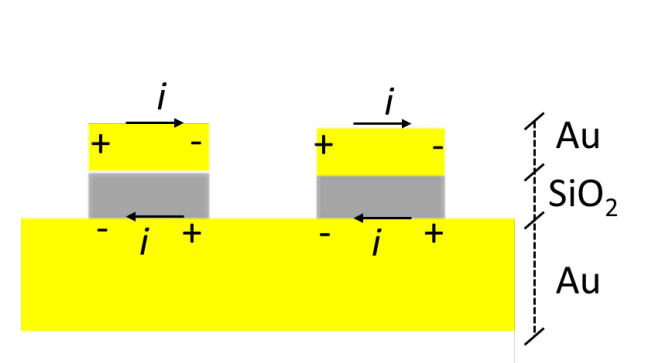

(a)

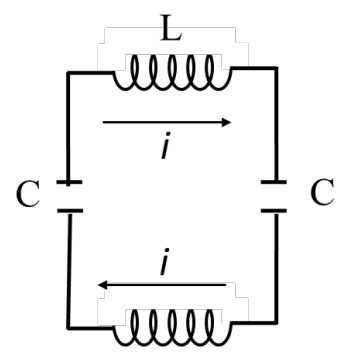

$\mathrm{L}$

(b)

Fig. 2. (a) Schematic drawing of the current distribution at the resonant frequency and (b) an equivalent circuit model for the single-band MMA.

Due to the coupling between the MMA and the incident time-dependent magnetic field, the induced currents are antiparallel, yielding a Lorentz-like magnetic response. Many studies 
have pointed out that the absorption behavior of MMAs is associated with the magnetic resonant loss and the magnetic resonant frequency therefore is used to determine the absorption frequency. The antiparallel distribution of charge currents in Fig. 2(a) can be transformed to an equivalent circuit as presented in Fig. 2(b). The metallic patterns and their images can be considered as inductors while the gap between them can be assigned as capacitors. The interactions between adjacent cells are about less than $10 \%$ of the intra-structure interactions and can be neglected. The magnetic resonant frequency hence can be estimated from the inductance $L$ and the capacitance $C$ as in Ref. [22]:

$$
f=\frac{1}{2 \pi \sqrt{L C}}
$$

where

$$
\begin{gathered}
C=\frac{\alpha \varepsilon \pi d^{2}}{4 t_{s}}, \\
L=\frac{\mu \pi t_{s}}{4},
\end{gathered}
$$

with $\varepsilon$ and $\mu$ being the permittivity and permeability of the $\mathrm{SiO}_{2}$ spacer, respectively.

In Eq. (2) a geometrical factor $\alpha$ is introduced to determine the effective surface area where induced charges accumulate at the top and bottom edges of the disks (rather than being distributed over the whole disk). For the disk-shaped structure, an estimated value of 0.235 for $\alpha$ has been employed showing the best fit between simulated and measured results. The disk inductance $L$ is obtained via the magnetic flux going through the dielectric volume.

\section{III.2. The absorption behavior of four-band MMAs}

The absorption frequencies calculated using Eqs. (1), (2) and (3) according to $d=1.2,1.5$, 1.8 and $2.0 \mu \mathrm{m}$ are listed in Table 1. The dielectric constant of $\mathrm{SiO}_{2}$ is chosen to be 1.7 for the best fitting [23].

Table 1. The inductance, capacitance, absorption frequencies, and frequency deviation calculated using Eqs. (1-3) according to $d=1.2,1.5,1.8$, and $2.0 \mu \mathrm{m}$ with a spacer thicknesses $t_{s}$ of $40 \mathrm{~nm}$. The frequency deviation is estimated from the difference between designed and experimental values of the disk diameter.

\begin{tabular}{|l|l|l|l|l|}
\hline $\boldsymbol{d}(\mu \mathrm{m})$ & $\boldsymbol{L}(\mathrm{H})$ & $\boldsymbol{C}(F)$ & $\boldsymbol{f}(\mathrm{THz})$ & $\Delta \boldsymbol{f}(\mathrm{THz})$ \\
\hline $1.2 \pm 0.1$ & $3.95 \times 10^{-14}$ & $9.21 \times 10^{-17}$ & 83.5 & $77.1-91.1$ \\
\hline $1.5 \pm 0.1$ & $3.95 \times 10^{-14}$ & $1.44 \times 10^{-16}$ & 66.8 & $62.6-71.5$ \\
\hline $1.8 \pm 0.1$ & $3.95 \times 10^{-14}$ & $2.07 \times 10^{-16}$ & 55.6 & $52.7-58.9$ \\
\hline $2.0 \pm 0.1$ & $3.95 \times 10^{-14}$ & $2.56 \times 10^{-16}$ & 50.1 & $47.7-52.7$ \\
\hline
\end{tabular}

In Table $1 f$ is the central frequency of the absorption peak and $\Delta f$ is the range of the resonant frequency $f$ which is calculated by the model when the diameter parameter is taken from $(d-1)$ to $(d+1)$ micrometers. The absorption behavior of the fabricated multi-band MMAs is then examined by the FTIR spectroscopy. Figure 3 presents the absorption spectrum of an MMA with $t_{s}=40 \mathrm{~nm}$. The dotted line indicated the corresponding calculated absorption frequencies listed in Table 1. The area of sparse patterns illustrates the frequency deviation estimated from the difference between designed and experimental values of the disk diameter. Taking the cause 
of frequency deviations, the calculated and experimental absorption behavior are in an excellent agreement. The experimental absorption spectrum shows four absorption peaks at 52,56, 66, and $81 \mathrm{THz}$ while the calculated counterparts are at 50.1, 55.6, 66.8, and $83.5 \mathrm{THz}$, respectively. The tendency of increasing disk diameter as decreasing absorption frequency has been well investigated by earlier numerical studies and again confirmed in our experiments [6].

It should be mentioned that the intensity of absorption peaks in Fig. 3 is low (less than $40 \%$ for most peaks). In order to improve the absorption efficiency, the dielectric thickness $t_{s}$ plays an important role. Previous investigation found that the absorption energy mainly dissipates inside the dielectric spacer in the form of dielectric losses [17-22]. Increasing the dielectric thickness can increase the loss volume and consequently the absorption intensity can be higher. In this work, the four-band MMAs are fabricated at three different $t_{s}$ as $40 \mathrm{~nm}, 80 \mathrm{~nm}$, and $150 \mathrm{~nm}$ using the e-beam evaporator at a controlled deposition rate of $1 \AA / \mathrm{s}$. Higher values of $t_{s}$ face up to a practical difficulty: the degradation of the photoresist layer under high temperatures for a long time results in damaged samples after lift-off. For thicker $\mathrm{SiO}_{2}$ layers, the practical issue should be taken into account.

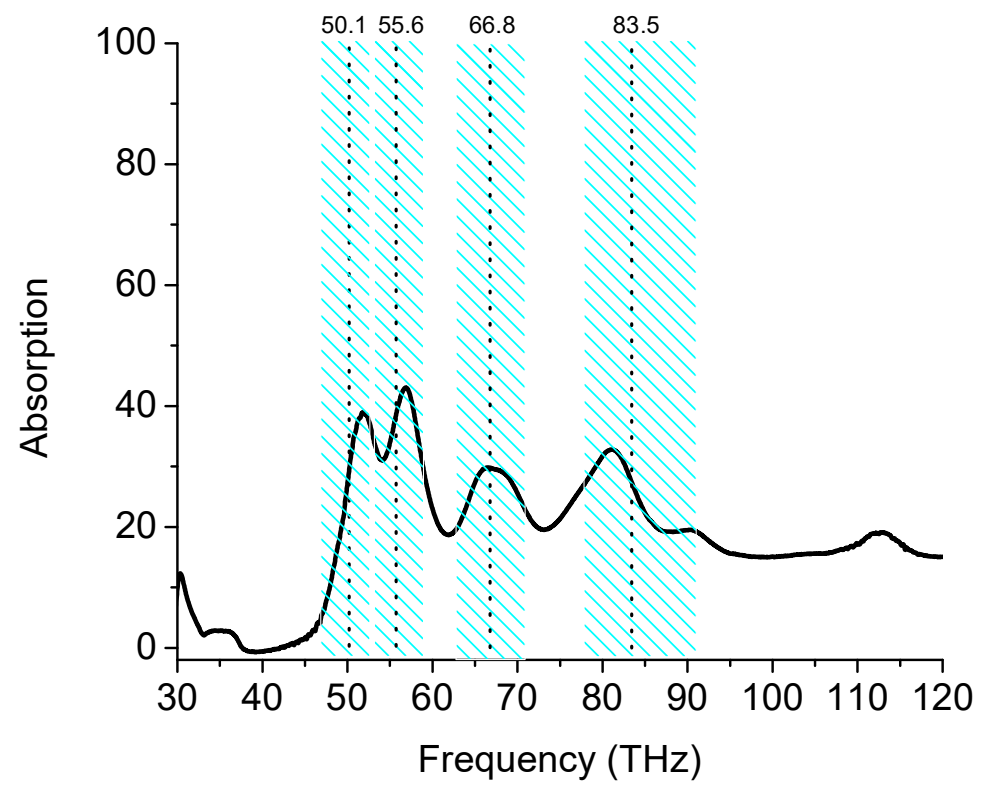

Fig. 3. Calculated absorption frequency in comparison with measured absorption spectra of four-band THz MMA with a spacer thicknesses $\mathrm{t}_{s}$ of $40 \mathrm{~nm}$.

The absorption spectra of MMAs with $t_{s}=40,80$ and $150 \mathrm{~nm}$ are presented in Fig. 4 . It appeared that the absorption intensity is significantly raising up with increasing $t_{s}$. In particular, the absorption peak corresponding to $d=1.8$ and $2.0 \mu \mathrm{m}$ increases from $40 \%$ at $t_{s}=40 \mathrm{~nm}$ to $70 \%$ at $t_{s}=80 \mathrm{~nm}$ and $90 \%$ at $t_{s}=150 \mathrm{~nm}$. The absorption peak corresponding to $d=1.2$ and $1.5 \mu \mathrm{m}$ increases from $30 \%$ at $t_{s}=40 \mathrm{~nm}$ to $50 \%$ at $t_{s}=80 \mathrm{~nm}$ and $70 \%$ at $t_{s}=150 \mathrm{~nm}$. The absorption peaks corresponding to disks with smaller diameters are weaker than those with bigger diameters. This can be attributed to the difference between filling factors of different-diameter 
disks. The bigger disks have higher filling factor and respond stronger to the excitation field while the smaller ones respond weaker, leading to lower absorption intensities. Of course, the possible influence of frequency-dependent dielectric constant of $\mathrm{SiO}_{2}$ should not be excluded. We also notice a considerable but reasonable band-broadening effect for increasing $t_{s}$, in line with previous experimental data $[24,25]$.

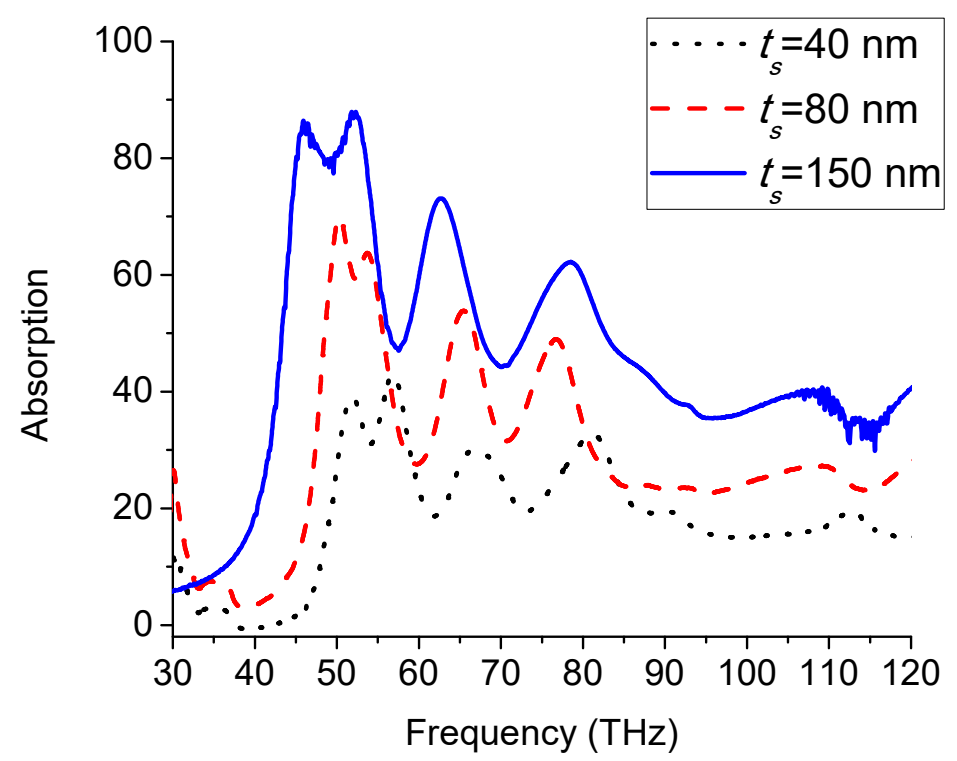

Fig. 4. Measured absorption spectra of four-band $\mathrm{THz}$ MMA with different $\mathrm{SiO}_{2}$ spacer thicknesses $t_{s}$.

\section{CONCLUSIONS}

In summary, we have designed, modeled, fabricated, and characterized a multi-band diskshaped MMA working at THz frequencies. The fabrication was carried out using the UV maskless lithography and e-beam evaporation technique and can be scaled up to centimeter-sized ranges. The absorption behavior was characterized by the micro-FTIR spectrometer. The equivalent circuit model was applied to understand the absorption mechanism and to predict the absorption frequency of the single-band and multi-band MMAs. It is demonstrated that the multi-band feature can be achieved by stacking different metamaterial resonators in a joint unit cell as theoretically anticipated. The thickness of the dielectric spacer was found to be vital in controlling the absorption strength. Our study indicated that increasing the dielectric thickness can considerably improve the absorption strength. The maximum thickness of the dielectric $\mathrm{SiO}_{2}$ layer obtained under our experimental conditions is $150 \mathrm{~nm}$ without thermally damaging the photoresist coating. This experimental observation would be useful for understanding the multi-band and broadband behavior of numerically designed MMAs. We believe that the obtained results can be applied for diversified applications using MMAs in the far-infrared and visible region. 


\section{ACKNOWLEDGEMENTS}

This work is supported by National Foundation for Science and Technology Development under grant number FWO.103.2017.01. NTT would like to thank Prof. Takuo Tanaka at RIKEN, Japan for his support in using experimental facilities.

\section{REFERENCES}

[1] Carlo Sirtori, Nature 417 (2002) 132.

[2] Gwyn P Williams, Rep. Prog. Phys. 69 (2006) 301.

[3] W. Xu, L. Xie, and Y. Ying, Nanoscale 9 (2017) 13864.

[4] N. T. Tung, Y. P. Lee, and V. D. Lam, Opt. Rev. 16 (2009) 578.

[5] N. T. Tung, T. X. Hoai, V. D. Lam, and Y. P. Lee, Comput. Mat. Sci. 49 (2010) 284.

[6] N. T. Tung and T. Tanaka, Photon. Nanostruct.: Fund. Appl. 28 (2018) 100.

[7] M. Lapke, T. Mussenbrock, R. P. Brinkmann, C. Scharwitz, M. Boke and J. Winter, Appl. Phys. Lett. 90 (2007) 121502.

[8] J. Huo, L. Wang and H. Yu, J. Mater. Sci. 44 (2009) 3917.

[9] N. I. Landy, S. Sajuyigbe, J. J. Mock, D. R. Smith and W. J. Padilla, Phys. Rev. Lett. 100 (2008) 207402.

[10] A. Ishikawa and T. Tanaka, Sci. Reports 5 (2015) 12570.

[11] C. M. Watts, X. Liu, W. J. Padilla, Adv. Mat. 24 (2012) OP98.

[12] Li Huang and Hou-Tong Chen, Terahertz Sci. Technol. 6 (2013) 26.

[13] N. T. Hien, B. S. Tung, N. T. Tuan, N. T. Tung, Y. P. Lee, N. M. An and V. D. Lam, Adv. Nat. Sci.: Nanosci. Nanotech. 5 (2014) 025013.

[14] D. H. Luu, N. V. Cuong, L. D. Hai, N. H. Tung, T. M. Cuong, L. D. Tuyen and V. D. Lam, J. Nonlinear Opt. Phys. Mater. 26 (2017) 1750036.

[15] T. M. Cuong, L. D. Hai, P. V. Hai, D. H. Tung, L. D. Tuyen, D. H. Luu and V. D. Lam, Sci. Rep. 8 (2018) 9523.

[16] B. X. Wang, Y. H. He, P. C. Lou and W. H. Xing, Nanoscale Adv. 2 (2020) 763.

[17] T. V. Huynh, B. X. Khuyen, B. S. Tung, S. T. Ngo, V. D. Lam, and N. T. Tung, Comput. Mat. Sci. 166 (2019) 276.

[18] U. T. D. Thuy, N. T. Thuy, N. T. Tung, E. Janssens, and N. Q. Liem, APL Mater. 7 (2019) 071102.

[19] P. T. Trang, B. H. Nguyen, D. H. Tiep, L. M. Thuy, V. D. Lam, and N. T. Tung, J. Elec. Mater. 45 (2016) 2547.

[20] B. S. Tung, N. V. Dung, B. X. Khuyen, N. T. Tung, P. Lievens, Y. P. Lee, and V. D. Lam, J. Opt. 15 (2013) 075101.

[21] N. T. Hien, L. N. Le, P. T. Trang, B. S. Tung, N. D. Viet, P. T. Duyen, N. M. Thang, D. T. Viet, Y. P. Lee, V. D. Lam, and N. T. Tung, Comp. Mat. Sci. 103 (2015) 189.

[22] L. N. Le, N. M. Thang, L. M. Thuy and N. T. Tung, Opt. Comm. 383 (2016) 244.

[23] C. Z. Tan, J. Non-Cryst. Solids 223 (1998) 158.

[24] V. D. Lam, N. T. Tung, M. H. Cho, J. W. Park, W. H. Jang and Y. P. Lee, J. Phys. D: Appl. Phys. 42 (2009) 115404.

[25] N. T. Tung, V. D. Lam, M. H. Cho, J. W. Park, W. H. Jang, and Y. P. Lee, Photon. Nanostruct.: Fund. Appl. 7 (2009) 206. 